Bull. Korean Math. Soc. 51 (2014), No. 4, pp. 1145-1153

http://dx.doi.org/10.4134/BKMS.2014.51.4.1145

\title{
THE AUTOMORPHISM GROUP OF COMMUTING GRAPH OF A FINITE GROUP
}

\author{
Mahsa Mirzargar, Peter P. Pach, and A. R. Ashrafi
}

\begin{abstract}
Let $G$ be a finite group and $X$ be a union of conjugacy classes of $G$. Define $C(G, X)$ to be the graph with vertex set $X$ and $x, y \in X$ $(x \neq y)$ joined by an edge whenever they commute. In the case that $X=$ $G$, this graph is named commuting graph of $G$, denoted by $\Delta(G)$. The aim of this paper is to study the automorphism group of the commuting graph. It is proved that $\operatorname{Aut}(\Delta(G))$ is abelian if and only if $|G| \leq 2$; $|\operatorname{Aut}(\Delta(G))|$ is of prime power if and only if $|G| \leq 2$, and $|\operatorname{Aut}(\Delta(G))|$ is square-free if and only if $|G| \leq 3$. Some new graphs that are useful in studying the automorphism group of $\Delta(G)$ are presented and their main properties are investigated.
\end{abstract}

\section{Introduction}

In this section we recall some definitions that will be used in the paper. Let $G$ be a group and $X$ a union of its conjugacy classes. Then $C(G, X)$ is the graph whose vertex set is $X$ with $x, y \in X, x \neq y$, joined by an edge whenever they commute. The graph $C(G, X)$ has been considered in many different ways, by several authors. The case $X=G \backslash Z(G)$ is denoted by $\Gamma(G)$, the complement of this graph has been studied in $[1,10]$. This graph has long been an object of interest in group theory $[7,11]$. We investigated the metric structure of the complement of $\Gamma(G)$, for finite groups, [8]. In the case that $X=G$, this graph is called a commuting graph of $G$ and denoted by $\Delta(G)$.

We consider simple graphs which are undirected, without loops or multiple edges. For any graph $\Gamma$, we denote the sets of the vertices and the edges of $\Gamma$ by $V(\Gamma)$ and $E(\Gamma)$, respectively. Suppose $v \in V(\Gamma)$ and $V_{1}(\Gamma) \subseteq V(\Gamma)$. Then $N(v)$ is the set of neighbors of $v$ and $\left\langle V_{1}(\Gamma)\right\rangle$ is the subgraph of $\Gamma$ induced by $V_{1}(\Gamma)$. The complement of $\Gamma$ is the graph $\bar{\Gamma}$ on the same vertices such that two vertices of $\bar{\Gamma}$ are adjacent if and only if they are not adjacent in $\Gamma$. For two graphs with disjoint vertex sets $V_{1}$ and $V_{2}$ their union is the graph $H$ in which $V(H)=V_{1} \cup V_{2}$ and $E(H)=E_{1} \cup E_{2}$. Define $n H$ to be the union of $n$ disjoint

Received September 12, 2013.

2010 Mathematics Subject Classification. 20B25.

Key words and phrases. commuting graph, automorphism group, extra special group. 
copies of $H$. The set of all automorphisms of a graph $\Gamma$ forms a permutation group, Aut $(\Gamma)$, acting on the object set $V(\Gamma)$.

Let $A$ and $B$ be permutation groups acting on object sets $X$ and $Y$, respectively. Define $B \nmid A=\{(a, f) \mid a \in A, f: X \rightarrow B\},(a, f)(x, y)=\left(a x, b_{x} y\right)$ where $f(x)=b_{x}$. B $\{A$ is called the wreath product. It acts on $X \times Y$ as follows: for each $a \in A$ and any sequence $b_{1}, b_{2}, \ldots, b_{n}$ (where $n=|X|$ ) in $\mathrm{B}$, there is a unique permutation in $A$ \ $B$ written $\left(a ; b_{1}, \ldots, b_{n}\right)$, and $\left(a ; b_{1}, \ldots, b_{n}\right)\left(x_{i}, y_{i}\right)=$ $\left(a x_{i}, b_{i} y_{i}\right)$.

Suppose $S_{n}$ denotes the symmetric group on $\{1,2, \ldots, n\}$. In what follows, we describe some important results relating the automorphism group of a graph which are crucial in this paper. Frucht [4] described if $\Gamma$ is a connected graph, then $\operatorname{Aut}(n \Gamma) \cong(\operatorname{Aut}(\Gamma)) 2 S_{n}$, by [3], if no component of $\Gamma_{1}$ is isomorphic with a component of $\Gamma_{2}$, then $\operatorname{Aut}\left(\Gamma_{1} \cup \Gamma_{2}\right) \cong \operatorname{Aut}\left(\Gamma_{1}\right) \times \operatorname{Aut}\left(\Gamma_{2}\right)$ and applying the last two theorems we have the result: Let $\Gamma=n_{1} \Gamma_{1} \cup n_{2} \Gamma_{2} \cup \cdots \cup n_{r} \Gamma_{r}$, where $n_{i}$ is the number of components of $\Gamma$ isomorphic to $\Gamma_{i}$, then

$\operatorname{Aut}(\Gamma) \cong\left(\left(\operatorname{Aut}\left(\Gamma_{1}\right)\right)<S_{n_{1}}\right) \times\left(\left(\operatorname{Aut}\left(\Gamma_{2}\right)\right)<S_{n_{2}}\right) \times \cdots \times\left(\left(\operatorname{Aut}\left(\Gamma_{r}\right)\right)<S_{n_{r}}\right)$.

Throughout this paper our notation is standard and taken mainly from $[2,12,14]$.

\section{Main result}

The commuting graphs were important in the 1960 s and 70 s as they provided early constructions of the modern sporadic simple groups and played an important role in the classification of $\{2,3\}$-transposition groups. Since then, various authors have continued to study these graphs determining many combinatorial quantities associated with graphs such as their diameter and their clique number and giving a similar treatment to closely related graphs such as the commuting involution graphs. With the best of our knowledge there is noting in literature that considered the automorphism group structure on commuting or non-commuting graphs.

Lemma 1. Let $X$ be a subset of $G$ of size $k$ such that $C_{G}(x)=C_{G}(y)$ for all $x, y \in X$. Then Aut $(\Delta(G))$ contains a symmetric group $S_{k}$ that acts $k$ transitively on $X$ while fixing $G \backslash X$ point-wise. We stress three specific cases we shall need:

(i) $X=Z(G)$;

(ii) $X=\left\{x^{i} \mid(i, n)=1\right\}$, where $x \in G$ has order $n$ (in which case $|X|=$ $\phi(n))$

(iii) $X=\left\{x, z x, x^{-1}, z x^{-1}\right\}$, where $x \in G, z \in Z(G)$ (in which case $|X|=4$ provided $z \neq 1$ and $\left.x^{2} \notin\{1, z\}\right)$.

Proof. Obvious.

Lemma 2. Let $|G| \geq 3$ and $|Z(G)|=2$. Then $\operatorname{Aut}(\Delta(G))$ is nonabelian, and $|\operatorname{Aut}(\Delta(G))|$ is neither a prime power nor square-free. 
Proof. If there exists $x \in G$ such that $x^{2} \notin Z(G)$, then we may apply Lemma 1(iii) to obtain an $S_{4}$ in $\operatorname{Aut}(\Delta(G))$, and the result follows. Thus we assume $x^{2} \in Z(G)$ for all $x \in G$. But this means $\frac{G}{Z(G)}$ is an elementary abelian 2group, i.e., $\mathrm{G}$ is extraspecial of order $2^{2 m+1}$ for some $m \geq 1$ [15]. If $m=1$, then $G \cong D_{8}$ or $Q_{8}$ and we have $\operatorname{Aut}(\Delta(G)) \cong\left(Z_{2} 2 S_{3}\right) \times Z_{2}$ and $|\operatorname{Aut}(\Delta(G))|=864$, as desired. If $m>2$, then $\frac{\operatorname{Aut}(G)}{\operatorname{Inn}(G)}$ is isomorphic to one of the two orthogonal groups $O^{ \pm}(2 m ; 2)$ and by $[6$, Table I] these are not prime power or square-free order group. This completes the proof.

Lemma 3. Let $G \neq 1$ be a finite group with $Z(G)=1$. Then

(i) $\operatorname{Aut}(\Delta(G))$ is non-abelian,

(ii) $|\operatorname{Aut}(\Delta(G))|$ is not a prime power,

(iii) $|\operatorname{Aut}(\Delta(G))|$ is not square-free.

Proof. Since $G$ is centerless, we see at once that $G$ cannot be abelian, nor can it be a $p$-group for any prime $p$. Moreover, $G$ embeds in $\operatorname{Aut}(\Delta(G))$ in this case, whence (i) and (ii) both follow. Suppose that $|\operatorname{Aut}(\Delta(G))|$ is square-free, in which case $|G|$ is square-free as well. By Lemma $1($ ii), we have $\pi(G)=\{2,3\}$. Thus, since $G$ is non-abelian, we have $G \cong S_{3}$. But $\operatorname{Aut}\left(\Delta\left(S_{3}\right)\right) \cong S_{3} \times Z_{2}$, which establishes (iii).

We are now ready to prove one of our main results as follows:

Theorem 1. Let $G$ be a finite group. Then the following are hold:

(i) $\operatorname{Aut}(\Delta(G))$ is abelian if and only if $|G| \leq 2$,

(ii) $|\operatorname{Aut}(\Delta(G))|$ is a prime power if and only if $|G| \leq 2$,

(iii) $|\operatorname{Aut}(\Delta(G))|$ is square-free if and only if $|G| \leq 3$.

Proof. Since $\operatorname{Aut}\left(\Delta\left(Z_{2}\right)\right) \cong Z_{2}$ and $\operatorname{Aut}\left(\Delta\left(Z_{3}\right)\right) \cong S_{3}$, all statements are verified in the reverse direction. To establish these statements in the forward direction, we may assume $Z(G) \neq 1$ by virtue of Lemma 3. First assume that $\operatorname{Aut}(\Delta(G))$ is either abelian or a p-group. As Aut $(\Delta(G))$ cannot contain $S_{3}$, we conclude from Lemma $1($ i) that $|Z(G)|=2$. The result now follows from Lemma 2. To establish (iii), we assume that $|\operatorname{Aut}(\Delta(G))|$ is square-free. Then $|Z(G)| \leq 3$ follows at once from Lemma $1(i)$, as does $\pi(G)=\{2,3\}$ from Lemma 1 (ii). Hence, as $\frac{G}{Z(G)} \cong \operatorname{Inn}(G)$ must also have square-free order, it is clear that $\left|\frac{G}{Z(G)}\right|$ divides 6 . Suppose that $\frac{G}{Z(G)} \cong S_{3}$. Let $\theta \in \operatorname{Aut}(\Delta(G))$ interchange 1 and $z$ and fix all remaining vertices of $\Delta(G)$, where $Z(G)=\langle z\rangle$. Then $\left\langle\theta, \iota_{x}\right\rangle \cong Z_{2} \times Z_{2}$, where $\iota_{x}$ is any involution $\operatorname{in} \operatorname{Inn}(G)$. Thus 4 divides $|\operatorname{Aut}(\Delta(G))|$, and we reach a contradiction. This proves that $\frac{G}{Z(G)}$ must be cyclic, whence $G$ is abelian, and the proof now follows since $|G|=|Z(G)| \leq 3$.

Lemma 4. Let $G$ be a finite group. Then

(1) $\operatorname{Aut}(G)=\operatorname{Aut}(\Delta(G))$ if and only if $|G|=1$. 
(2) $\operatorname{Aut}(\Delta(G)) \cong \operatorname{Aut}(\Gamma(G)) \times S_{|Z(G)|}$.

Proof. (1) It is easily seen that the map $\phi: x \rightarrow x^{-1}$ is an automorphism of the graph $\Delta(G)$, but it is an automorphism of the group $G$ if and only if $G$ is abelian. If $\operatorname{Aut}(G)=\operatorname{Aut}(\Delta(G))$ and $|G|=n$, then $G$ is abelian and $\operatorname{Aut}(G)=\operatorname{Aut}(\Delta(G)) \cong S_{n}$. We conclude that $|G|=1$.

(2) Suppose $\sigma \in S_{|Z(G)|}$. Define $A=\{f \in \operatorname{Aut}(\Delta(G)) \mid \forall z \in Z(G) ; f(z)=$ $z\}$ and $B=\left\{f_{\sigma} \mid \sigma \in S_{|Z(G)|}\right\}$, where $f_{\sigma}: G \longrightarrow G$ is defined by

$$
f_{\sigma}(x)= \begin{cases}x & x \notin Z(G) \\ \sigma(x) & x \in Z(G) .\end{cases}
$$

Then by the definition of $\Gamma(G)$ and $\Delta(G)$, Aut $(\Gamma(G)) \cong A \unlhd \operatorname{Aut}(\Delta(G)), B \unlhd$ $\operatorname{Aut}(\Delta(G))$ and $A \cap B=1$. Since $\operatorname{Aut}(\Delta(G))=\langle A \cup B\rangle$, Aut $(\Delta(G)) \cong A \times B \cong$ $\operatorname{Aut}(\Gamma(G)) \times S_{|Z(G)|}$, proving the result.

Suppose that $G$ is a finite group. Then it is clear that $\operatorname{Aut}(\Gamma(G))$ and $\operatorname{Aut}(G)$ are subgroups of $\operatorname{Aut}(\Delta(G))$. Define two permutations $\Phi_{x, y}, \phi: G \rightarrow G$ as follows: $\Phi_{x, y}=(x, y)$ and the permutation $\phi$ is inverse mapping $x \rightarrow x^{-1}$. We also define $\operatorname{Aut}^{*}(G)=\langle\operatorname{Aut}(G), \phi\rangle$ and restrict our attention to the equality of the subgroup and the main group.

Theorem 2. $\operatorname{Aut}^{*}(G)=\operatorname{Aut}(\Delta(G))$ if and only if $G \cong S_{3}$.

Proof. We first notice that if $G \cong S_{3}$, then $\operatorname{Aut}\left(\Delta\left(S_{3}\right)\right)=\operatorname{Aut}\left(\Gamma\left(S_{3}\right)\right)$. Since $\Gamma\left(S_{3}\right)$ is a union of three isolated vertices and one edge, $\operatorname{Aut}\left(\Delta\left(S_{3}\right)\right) \cong Z_{2} \times S_{3}$. On the other hand, $\operatorname{Aut}^{*}\left(S_{3}\right)=\left\langle S_{3}, \phi\right\rangle \cong Z_{2} \ltimes S_{3}$, as desired.

Conversely, suppose that $\operatorname{Aut}^{*}(G)=\operatorname{Aut}(\Delta(G))$. We first prove $|Z(G)|=1$. If there exists $1 \neq a \in Z(G)$, then the map $f_{a}: G \rightarrow G, x \rightarrow a x$, is an element of $\operatorname{Aut}(\Delta(G))$. Since $\phi$ is an automorphism of order 2 and each $f \in \operatorname{Aut}(G)$ commute with $\phi, f_{a}=f_{1} \circ \phi$, where $f_{1} \in \operatorname{Aut}(G)$. By definition of $f_{a}$ and $\phi$ it can easily seen that for each $x \in G, f_{1}(x)=x^{-1} a^{-1}$, a contradiction. Therefore $|Z(G)|=1$. Consider an element $x \in G$ and define $\psi_{x}: G \rightarrow G$ which sends the elements $x, x^{-1} \in G$ to their inverses and assigns each element of $G \backslash\left\{x, x^{-1}\right\}$ to itself. We claim that $\psi_{x} \in \operatorname{Aut}(\Delta(G)) \backslash \operatorname{Aut}(G)$, when $o(x)>2$. To do this, we notice that $\psi_{x} \in \operatorname{Aut}(G)$ if and only if $o(x)=2$. Suppose $\psi_{x} \in \operatorname{Aut}(G)$. Since $|Z(G)|=1$ and $G$ is a non-abelian group, $|G|>5$. Choose $t \in G \backslash\left\{1, x^{-2}\right\}$. Then $x t=\psi_{x}(x t)=\psi_{x}(x) \psi_{x}(t)=x^{-1} t$ and so $x^{2}=1$. Notice that if $G$ is an elementary abelian group of order $2^{m}$, then we have a contradiction by $|Z(G)|=1$. Now consider $x \in G$ with $o(x) \geq 3$. Since $\psi_{x} \in \operatorname{Aut}(\Delta(G))=$ Aut $^{*}(G), \psi_{x}=f \circ \phi$, where $f \in \operatorname{Aut}(G)$. Thus, $f(x)=x, f\left(x^{-1}\right)=x^{-1}$, and $f(y)=y^{-1}$ for $y \in G \backslash\left\{x, x^{-1}\right\}$. We claim that $G$ has exactly two elements of order three and other non-identity elements are involutions. If there exists $x \in G$ with $o(x) \geq 4$, then we choose $t \in G \backslash\left\{x, x^{-1}\right\}$ and consider the image of $x^{2} t$ under $f$. If $x^{2} t=x$, then $t=x^{-1}$, a contradiction. If $x^{2} t=x^{-1}$, then $x^{-1}=f\left(x^{2} t\right)=f\left(x^{2}\right) f(t)=x^{-2} t$ and $x=t$, which is impossible. Finally, $x^{2} t \neq x, x^{-1}$ and so $f\left(x^{2} t\right)=t^{-1} x^{-2}$ and $f\left(x^{2}\right) f(t)=x^{-2} t^{-1}$ which implies 
that $x^{-2} t^{-1}=t^{-1} x^{-2}$. The later shows that $x^{2} \in Z(G)=1$, leads to our final contradiction. Hence, $G$ does not have elements of order $\geq 4$.

On the other hand, if $o(x)=3$, then $f\left(x^{2} t\right)=f\left(x^{2}\right) f(t)$ and we have $t^{-1} x t=$ $x^{-1}$. Since $G$ is not an elementary abelian 3-group, $G$ has an element $z$ with $o(z)=2$. Suppose $G$ has an element $y \neq x, x^{-1}$ with $o(y)=3$. Then we have three choices for $f\left(y^{2} z\right)$. If $y^{2} z=x$, then $f\left(y^{2} z\right)=y^{2} z$ and $f\left(y^{2}\right) f(z)=y z$. Therefore, $y=1$ which is impossible. A similar argument shows that $y^{2} z$ cannot be equal to $x^{-1}$. If $f\left(y^{2} z\right)=z y$ and $f\left(y^{2}\right) f(z)=y z$, then $y \in C_{G}(z)$ which implies that $o(y z)=6$, a contradiction. Therefore, $\left\{x, x^{-1}\right\}$ is the unique $G$-conjugacy class of elements of order 3 .

Our discussion above shows that $G=\left\langle x, t_{1}, t_{2}, \ldots, t_{n}\right\rangle$ such that $o\left(t_{i}\right)=2$, $o(x)=3$ and $t_{i}^{-1} x t_{i}=x^{-1}$ where $1 \leq i \leq n$. We have two cases:

1) If for $1 \leq i \neq j \leq n, t_{i} t_{j}=t_{j} t_{i}$ and $n \geq 2$, then $t_{1}^{-1} x t_{1}=x^{-1}$ and $t_{2}^{-1} x t_{2}=x^{-1}$. So $\left(t_{1} t_{2}\right)^{-1} x\left(t_{1} t_{2}\right)=x$ or $\left(t_{1} t_{2}\right) x=x\left(t_{1} t_{2}\right)$. Hence $t_{1} t_{2} \in Z(G)$, which is a contradiction. Therefore $n=1$ and $G \cong S_{3}$.

2) Suppose $t_{1} t_{2} \neq t_{2} t_{1}$ then $o\left(t_{1} t_{2}\right)=3, t_{1} t_{2}=x$ and $t_{2} t_{1}=x^{-1}$. If for every $t_{i}, t_{i} t_{1}=t_{1} t_{i}$ and $t_{i} t_{2}=t_{2} t_{i}$, then $x^{-1}=t_{i}^{-1} x t_{i}=t_{i}^{-1}\left(t_{1} t_{2}\right) t_{i}=t_{1} t_{2}=x$, which is a contradiction. So, $t_{i} t_{2}=x^{-1}$ and $t_{2} t_{i}=x$. If $t_{i} t_{1}=t_{1} t_{i}$, then $x^{-1}=t_{1}^{-1} x t_{1}=t_{1}^{-1} t_{2} t_{i} t_{1}=t_{1}^{-1} t_{2} t_{1} t_{i}=t_{1}^{-1} x^{-1} t_{i}=x t_{1} t_{i}$, therefore $t_{1} t_{i}=x$ which is impossible. Finally, we consider the group $G=\left\langle t_{1}, t_{2}, t_{3}\right\rangle$ such that $t_{1} t_{2}=t_{2} t_{3}=t_{3} t_{1}=x$. According to the above, it is obvious that $G \cong S_{3}$. If $n \geq 4$, then $\left\{t_{1}, t_{2}, t_{3}\right\} \subseteq C_{G}\left(t_{4}\right)$ and so $G$ is isomorphic to $S_{3}$, as desired.

We consider the right cosets $Z(G) x_{1}, Z(G) x_{2}, \ldots, Z(G) x_{m-1}$ of the group $G$ and define a new graph $\Delta^{u}(G)$ with

$$
\begin{aligned}
& V\left(\Delta^{u}(G)\right)=\left\{x_{0}=1, x_{1}, \ldots, x_{m-1}\right\} \\
& E\left(\Delta^{u}(G)\right)=\left\{x_{i} x_{j} \mid x_{i} x_{j}=x_{j} x_{i}, 0 \leq i<j \leq m-1\right\} .
\end{aligned}
$$

Notice when $|Z(G)|=1$ then $\Delta(G) \cong \Delta^{u}(G)$ [9]. It is clear that every two elements in one of these cosets commute. Hence we have complete graph in any of these cosets. On the other hand, if there exist $x_{i} \in Z(G) x_{i}, x_{j} \in Z(G) x_{j}$ satisfying $x_{i} x_{j}=x_{j} x_{i}$, then for every $y_{i} \in Z(G) x_{i}, y_{j} \in Z(G) x_{j}$ we have $y_{i} y_{j}=y_{j} y_{i}$. Finally, the set of all $\phi \in \operatorname{Aut}(\Delta(G))$ such that for all $a, b \in G$, $a b^{-1} \in Z(G)$ implies that $\phi(a) \phi(b)^{-1} \in Z(G)$ is denoted by $T$.

Theorem 3. Let $G$ be a finite group. Then,

(1) $\operatorname{Aut}\left(\Delta^{u}(G)\right)$ is a subgroup of $\operatorname{Aut}(\Delta(G))$. Moreover, $\operatorname{Aut}\left(\Delta^{u}(G)\right)=$ $\operatorname{Aut}(\Delta(G))$ if and only if $|Z(G)|=1$.

(2) If $G$ is not centerless, then $T$ is a subgroup of $\operatorname{Aut}(\Delta(G))$, and $\operatorname{Aut}(\Delta(G))=T$ if and only if for each pair $a, b$ of elements of $G$ with $C_{G}(a)=C_{G}(b)$, we have $a b^{-1} \in Z(G)$.

Proof. (1) Suppose for every $0 \leq i \leq m-1, Z(G) x_{i}=\left\{x_{i, 1}, \ldots, x_{i,|Z(G)|}\right\}$. For every $\phi \in \operatorname{Aut}\left(\Delta^{u}(G)\right)$ we define the automorphism $\phi^{\prime} \in \operatorname{Aut}(\Delta(G))$ in this way: $\phi^{\prime}\left(x_{i, k}\right)=x_{j, k}$, where $\phi\left(x_{i}\right)=x_{j}$. The set of all such automorphisms is 
isomorphic to $\operatorname{Aut}\left(\Delta^{u}(G)\right)$ and also this set is a subgroup of $\operatorname{Aut}(\Delta(G))$. If $\operatorname{Aut}\left(\Delta^{u}(G)\right)=\operatorname{Aut}(\Delta(G))$ and $x_{0,1}, x_{0,2} \in Z(G)$, then $\Phi_{x_{0,1}, x_{0,2}} \in \operatorname{Aut}(\Delta(G))$ but not in $\operatorname{Aut}\left(\Delta^{u}(G)\right)$. Therefore $|Z(G)|=1$. The converse of equality is trivial.

(2) It is enough to prove that if $\phi_{1}, \phi_{2} \in T$, then $\phi_{1} \circ \phi_{2} \in T$. Suppose $a b^{-1} \in Z(G)$ and $Z(G) \phi_{1}(a)=Z(G) \phi_{1}(b)$ then $Z(G) \phi_{1} \circ \phi_{2}(a)=Z(G) \phi_{1} \circ$ $\phi_{2}(b)$ as desired. For equality suppose $\operatorname{Aut}(\Delta(G))=T, a, b \in G$ such that $C_{G}(a)=C_{G}(b)$ and $a b^{-1} \notin Z(G)$. Since $|Z(G)|>1$, there is an element $a^{\prime} \in Z(G) a$ such that $a \neq a^{\prime}$. If $\phi_{a^{\prime}, b} \in \operatorname{Aut}(\Delta(G))$, then $a a^{\prime-1} \in Z(G)$ and $\phi(a) \phi\left(a^{\prime}\right)^{-1} \notin Z(G)$, leads to a contradiction. Suppose $\phi \in \operatorname{Aut}(\Delta(G))$ and $a, b \in G$ satisfying $a b^{-1} \in Z(G)$ and $C_{G}(a)=C_{G}(b)$ then $C_{G}(\phi(a))=C_{G}(\phi(b))$ and by assumption we obtain that $\phi(a) \phi(b)^{-1} \in Z(G)$. Therefore $\phi \in T$.

Corollary 1. Let $|Z(G)| \geq 2$, where $G$ be a non-abelian group. If $T=$ $\operatorname{Aut}(\Delta(G))$, then $G / Z(G)$ is an elementary abelian 2-group.

Proof. By Theorem 3, for every $x \in G, x^{2} \in Z(G)$. Therefore $G / Z(G)$ is an elementary abelian 2-group.

Suppose $\left|\frac{G}{Z(G)}\right|=4$, then $T=\operatorname{Aut}(\Delta(G))$ if and only if $\Delta^{u}(G)$ is a star. So, it is natural to ask the following question:

Question. What can be the graph $\Delta^{u}(G)$ when $T=\operatorname{Aut}(\Delta(G))$ and $\frac{G}{Z(G)}$ is an elementary abelian group of order $2^{n}$, for $n>2$ ?

For a finite group $G$ we define a labeled graph $\Delta^{v}(G)$ as follows. For $a, b \in$ $G$ let $a \sim b$ if $C_{G}(a)=C_{G}(b)$. Clearly, $\sim$ is an equivalence relation, the equivalence class of $a \in G$ is $A(a)=\left\{x \mid C_{G}(x)=C_{G}(a)\right\}$. Let us denote the equivalence classes by $A_{1}, \ldots, A_{k}$, these are the vertices of $\Delta^{v}(G)$. Two vertices $A_{i}$ and $A_{j}$ are connected if and only if $a_{i} a_{j}=a_{j} a_{i}$ for some $a_{i} \in$ $A_{i}, a_{j} \in A_{j}$. At first we note that if there exist $a_{i} \in A_{i}, a_{j} \in A_{j}$ satisfying $a_{i} a_{j}=a_{j} a_{i}$, then for every $b_{i} \in A_{i}, b_{j} \in A_{j}$ we have $a_{j} \in C_{G}\left(a_{i}\right)=C_{G}\left(b_{i}\right)$. So, $b_{i} \in C_{G}\left(a_{j}\right)=C_{G}\left(b_{j}\right)$ implies that $b_{i} b_{j}=b_{j} b_{i}$. Each equivalence class is the union of some sets of the form $t Z(G)$, hence there exists a positive integers $c_{i}$ such that $\left|A_{i}\right|=c_{i}|Z(G)|$. Let $\alpha\left(A_{i}\right)=c_{i}$ be the label of the vertex $A_{i}$ in $\Delta^{v}(G)$. We say that $\phi: V\left(\Delta^{v}(G)\right) \rightarrow V\left(\Delta^{v}(G)\right)$ is an automorphism of the labeled graph $\Delta^{v}(G)$ if $\phi$ is a bijection, it preserves the edges (and the nonedges) and it preserves the labels. The automorphism group formed by these automorphisms is denoted by $\operatorname{Aut}\left(\Delta^{v}(G)\right)$.

Define $S_{A_{i}}=\left\{f_{\sigma} \mid \sigma \in S_{\left|A_{i}\right|}, \forall x \in A_{i}, f_{\sigma}(x)=\sigma(x), \forall x \notin A_{i}, f_{\sigma}(x)=x\right\}$, $1 \leq i \leq k$. Clearly, $S_{A_{i}}$ is a subgroup of $\operatorname{Aut}(\Delta(G))$. The connection between $\operatorname{Aut}(\Delta(G))$ and $\operatorname{Aut}\left(\Delta^{v}(G)\right)$ is described by the following theorem:

Theorem 4. There is a subgroup $A$ of $\operatorname{Aut}(\Delta(G))$ such that $A \cong \operatorname{Aut}\left(\Delta^{v}(G)\right)$ and $\operatorname{Aut}(\Delta(G))=\left\langle S_{A_{1}}, \ldots, S_{A_{k}}\right\rangle \rtimes A$. 
Proof. Suppose $A_{i}=\left\{a_{i, 1}, a_{i, 2}, \ldots, a_{i,\left|A_{i}\right|}\right\}, 1 \leq i \leq k$. For each $f \in \operatorname{Aut}\left(\Delta^{v}(G)\right)$, define $\bar{f}\left(a_{i, r}\right)=a_{j, r}$ such that $f\left(A_{i}\right)=A_{j}, 1 \leq i, j \leq k, 1 \leq r \leq\left|A_{i}\right|$. Then $\bar{f} \in \operatorname{Aut}(\Delta(G))$. Set $A$ to be the set of all such automorphisms. Clearly, $A \cong \operatorname{Aut}\left(\Delta^{v}(G)\right)$ and $A$ is a subgroup of $\operatorname{Aut}(\Delta(G))$. We prove for every $f \in \operatorname{Aut}(\Delta(G)), f$ is a composition of some automorphisms of $A$ and $S_{A_{i}}$, $1 \leq i \leq k$. Define $\psi: V\left(\Delta^{v}(G)\right) \rightarrow V\left(\Delta^{v}(G)\right)$, such that $\psi\left(A_{i}\right)=f\left(A_{i}\right)$, for each $i$. Note that if $f\left(a_{i, r}\right) \in A_{j}$, then $f\left(A_{i}\right)=A_{j}$, because for each $b \in A_{i}$, $C_{G}(b)=C_{G}\left(a_{i, r}\right)$ and $C_{G}(f(b))=C_{G}\left(f\left(a_{i, r}\right)\right)$. So, $f(b) \in A_{j}$ and $\psi$ is an element of Aut $\left(\Delta^{v}(G)\right)$, because if $A_{r} A_{s}$ is an edge of $\Delta^{v}(G)$, then there exist $a_{r} \in A_{r}$ and $a_{s} \in A_{s}$ such that $a_{r} a_{s}$ is an edge of $\Delta(G)$. Thus, $f\left(a_{r}\right) f\left(a_{s}\right) \in$ $E(\Delta(G))$. We conclude that $\psi\left(A_{r}\right) \psi\left(A_{s}\right)=f\left(A_{r}\right) f\left(A_{s}\right) \in E\left(\Delta^{v}(G)\right)$. On the other hand, $\psi$ preserves the labels, because $\left|A_{i}\right|=\left|f\left(A_{i}\right)\right|=\left|\psi\left(A_{i}\right)\right|$. Therefore $\psi \in \operatorname{Aut}\left(\Delta^{v}(G)\right)$. Suppose $\bar{\psi} \in A$ corresponds to $\psi$. If $a_{i, r}$ is arbitrary and $f\left(a_{i, r}\right)=a_{j, s}$, then there exists $f_{\sigma_{j}} \in S_{A_{j}}$ such that $f_{\sigma_{j}}\left(a_{j, r}\right)=a_{j, s}$. For $1 \leq i \leq k$ we can choose $f_{\sigma_{i}} \in S_{A_{i}}$ such that $f=f_{\sigma_{1}} \circ f_{\sigma_{2}} \circ \cdots \circ f_{\sigma_{k}} \circ \bar{\psi}$. Therefore, $\operatorname{Aut}(\Delta(G))=\left\langle S_{A_{1}}, \ldots, S_{A_{k}}\right\rangle A$. Finally, for each $g \in \operatorname{Aut}(\Delta(G))$ and $f \in$ $\left\langle S_{A_{1}}, \ldots, S_{A_{k}}\right\rangle$, there exists $i, 1 \leq i \leq k$ such that $g^{-1} f g$ is a permutation on $A_{i}$ and we conclude that $\left\langle S_{A_{1}}, \ldots, S_{A_{k}}\right\rangle$ is a normal subgroup of $\operatorname{Aut}(\Delta(G))$. This completes our proof.

In the end of this paper the automorphism group of $\Delta(G)$ is computed, when $G$ is an AC group. To describe this concept, we first review some important properties of dihedral groups.

The dihedral group $D_{2 n}$, can be presented as follows:

$$
D_{2 n}=\left\langle x, y \mid x^{n}=y^{2}=1, y^{-1} x y=x^{-1}\right\rangle .
$$

If $n$ is odd, then $\left|Z\left(D_{2 n}\right)\right|=1, C_{D_{2 n}}\left(a^{i}\right)=\langle a\rangle$ and $C_{D_{2 n}}\left(a^{i} b\right)=\left\langle a^{i} b\right\rangle, 0 \leq i \leq$ $n-1$. If $n$ is even, then $Z\left(D_{2 n}\right)=\left\langle a^{\frac{n}{2}}\right\rangle, C_{D_{2 n}}\left(a^{i}\right)=\langle a\rangle, 1 \leq i \neq \frac{n}{2} \leq n-1$, and $C_{D_{2 n}}\left(a^{i} b\right)=\left\langle a^{i} b, a^{\frac{n}{2}}\right\rangle, 0 \leq i \leq n-1$. By above calculations, the graph $\Gamma\left(D_{2 n}\right)$ is:

- The union of complete graph $K_{n-1}$ and $n$ isolated vertices, when $n$ is odd. In this case, $\operatorname{Aut}\left(\Gamma\left(D_{2 n}\right)\right) \cong S_{n} \times S_{n-1}$.

- The union of complete graph $K_{n-2}$ and $\frac{n}{2}$ copies of $K_{2}$, which all vertices are adjacent by the elements of the center of $G$, when $n$ is even. In this case, $\operatorname{Aut}\left(\Gamma\left(D_{2 n}\right)\right) \cong S_{n-2} \times\left(S_{2}\left\langle S_{\frac{n}{2}}\right) \times S_{2}\right.$.

For the dihedral group, the map $\theta_{i, t}:\{x, y\} \rightarrow D_{2 n}$ such that $x \rightarrow x^{t}$ and $y \rightarrow x^{i} y, 0 \leq i \leq n-1,1 \leq t \leq n-1$ and $(t, n)=1$ can be extended to an automorphism $\gamma_{i, t}$ of $D_{2 n}$. Therefore, $\operatorname{Aut}\left(D_{2 n}\right)=\left\{\gamma_{i, t} \mid 0 \leq i \leq n-1,1 \leq t \leq\right.$ $n-1,(t, n)=1\}$. Thus $\left|\operatorname{Aut}\left(D_{2 n}\right)\right|=n \phi(n)$, where $\phi(n)$ is the Euler's totient function. On the other hand, we proved that $\left|\operatorname{Aut}\left(\Gamma\left(D_{2 n}\right)\right)\right|=n !(n-1)$ !, when $n$ is odd and $\left|\operatorname{Aut}\left(\Gamma\left(D_{2 n}\right)\right)\right|=(n-2) !\left(\frac{n}{2}\right) ! 2^{\frac{n}{2}+1}$, otherwise.

We say a group $G$ has abelian centralizers, if for each non-central element $x \in G$, the centralizer of $x$ in $G$ is abelian. A group $G$ is an AC-group, whenever the centralizers of non-central elements are abelian. The dihedral group $D_{2 n}$ 
is an example of an AC-group. In [13], Rocke proved that the following are equivalent:

(1) $G$ has abelian centralizers;

(2) If $x y=y x$, then $C_{G}(x)=C_{G}(y)$ whenever $x, y \notin Z(G)$;

(3) If $x y=y x$ and $x z=z x$, then $y z=z y$ whenever $x \notin Z(G)$;

(4) If $U$ and $B$ are subgroups of $G$ and $Z(G)<C_{G}(U) \leq C_{G}(B)<G$, then $C_{G}(U)=C_{G}(B)$.

Therefore, the intersection of two proper element centralizers of an AC-group is the center of $G$. If $G$ is an AC-group, then $\Delta(G)$ is a union of some complete graphs which all vertices are adjacent to the elements of $Z(G)$. So, $\Delta(G)$ is $n_{1}\left(C_{G}\left(x_{1}\right) \backslash Z(G)\right) \cup n_{2}\left(C_{G}\left(x_{2}\right) \backslash Z(G)\right) \cup \cdots \cup n_{r}\left(C_{G}\left(x_{r}\right) \backslash Z(G)\right)$ and also every elements of $Z(G)$ is adjacent to all elements of $G$, such that for each $i, 1 \leq i \leq r$, we have $n_{i}$ isomorphic components with complete graph of size $\left|C_{G}\left(x_{i}\right) \backslash Z(G)\right|$. Hence, we prove the following theorem:

Theorem 5. Let $G$ be an $A C$-group, with the above notation

$$
\begin{aligned}
\operatorname{Aut}(\Delta(G)) \cong & \left(\left(S_{\left|C_{G}\left(x_{1}\right)\right|-|Z(G)|}\right)\left\langle S_{n_{1}}\right) \times\left(\left(S_{\left|C_{G}\left(x_{2}\right)\right|-|Z(G)|}\right)<S_{n_{2}}\right) \times \cdots\right. \\
& \times\left(\left(S_{\left|C_{G}\left(x_{n}\right)\right|-|Z(G)|}\right)<S_{n_{r}}\right) \times S_{|Z(G)|} .
\end{aligned}
$$

Acknowledgement. The first author would like to thank the University of Kashan for supporting her visit from Department of Computer Science and Information Theory at Budapest University of Technology and Economics during which this work was done. She also would like to express her gratitude to professor G. Y. Katona for warm hospitality during her visit. The research of the first and third authors are partially supported by the University of Kashan under grant no 159020/16.

\section{References}

[1] A. Abdollahi, S. Akbari, and H. R. Maimani, Non-commuting graph of a group, J. Algebra 298 (2006), no. 2, 468-492.

[2] N. Biggs, Algebraic Graph Theory, Second ed., Cambridge Univ. Press, Cambridge, 1993.

[3] P. J. Cameron, Automorphisms of graphs, Beineke, Lowell W. (ed.) et al., Topics in Algebraic Graph Theory, Cambridge: Cambridge University Press, Encyclopedia of Mathematics and Its Applications 102 (2004), 137-155.

[4] R. Frucht, On the groups of repeated graphs, Bull. Amer. Math. Soc. 55 (1949), 418-420.

[5] The GAP Team, GAP, Groups, Algorithms and Programming, Lehrstuhl De für Mathematik, RWTH, Aachen, 1995.

[6] D. Gorenstein, R. Lyons, and R. Solomon, The Classification of the Finite Simple Groups, Mathematical Surveys and Monographs, 40.1. American Mathematical Society, Providence, RI, 1994.

[7] J. Lennox and J. Wiegold, Extension of a problem of Paul Erdös on groups, J. Aust. Math. Soc. Ser. A 31 (1981), no. 4, 459-463.

[8] M. Mirzargar and A. R. Ashrafi, Some distance-based topological indices of a noncommuting graph, Hacet. J. Math. Stat. 41 (2012), no. 4, 515-526.

[9] A. R. Moghaddamfar, On noncommutativity graphs, Siberian Math. J. 47 (2006), 911914. 
[10] A. R. Moghaddamfar, W. J. Shi, W. Zhou, and A. R. Zokayi, On the noncommuting graph associated with a finite group, Siberian Math. J. 46 (2005), no. 2, 325-332.

[11] B. H. Neumann, A problem of Paul Erdös on groups, J. Aust. Math. Soc. Ser. A 21 (1976), no. 4, 467-472.

[12] D. J. S. Robinson, A Course in the Theory of Groups, Springer-Verlag, New York, 1982.

[13] D. M. Rocke, p-groups with abelian centralizers, Proc. London Math. Soc. (3) 30 (1975), $55-75$.

[14] D. B. West, Introduction to Graph Theory, Prentice Hall. Inc. Upper Saddle River, NJ, 1996.

[15] D. L. Winter, The automorphism group of an extraspecial p-group, Rocky Mountain J. Math. 2 (1972), no. 2, 159-168.

Mahsa Mirzargar

Department of Pure Mathematics

Faculty of Mathematical Sciences

UNIVERSITY OF KASHAN

KASHAN 87317-51167, I. R. IRAN

E-mail address: m.mirzargar@gmail.com

Peter P. Pach

Department of Algebra and Number Theory

EÖTVÖS LORÁND UNIVERSITY

1117 Budapest, Pázmány Péter Sétány 1/C, Hungary

E-mail address: ppp24@cs.elte.hu, p.p.pach@gmail.com

A. R. Ashrafi

Department of Pure Mathematics

Faculty of Mathematical Sciences

UNIVERSITY OF KASHAN

KASHAN 87317-51167, I. R. IRAN

E-mail address: ashrafi@kashanu.ac.ir 